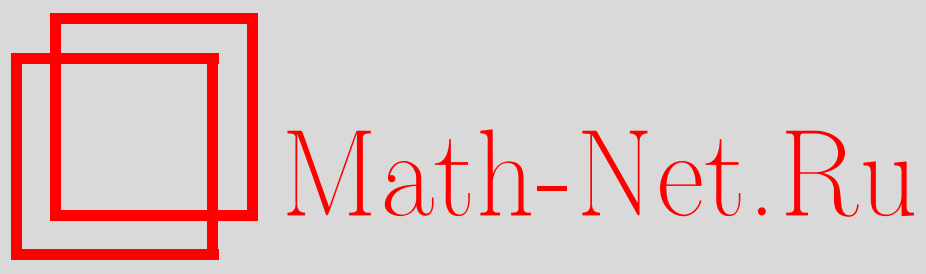

Е. И. Яшагин, Об одноточечных долях Глисона алгебр Бляшке, Функи. анализ и его прил., 2006, том 40, выпуск 1, 92-94

DOI: https://doi.org/10.4213/faa26

Использование Общероссийского математического портала Math-Net.Ru подразумевает, что вы прочитали и согласны с пользовательским соглашением

http://www.mathnet.ru/rus/agreement

Параметры загрузки:

IP : 54.80 .97 .219

26 апреля 2023 г., 12:53:09

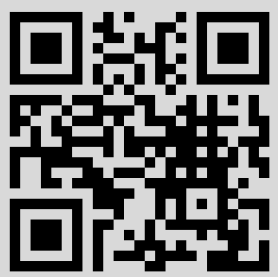




\section{Об одноточечных долях Глисона алгебр Бляшке}

(C) 2006. Е. И. ЯШАГИн

Введение. Пусть $A(X)$ - максимальная равномерная алгебра на компакте $X$. Если пространство $\operatorname{Re} A=\{\operatorname{Re} f: f \in A\}$ плотно в пространстве $C_{\mathbb{R}}(X)$ всех вещественнозначных функций на $X$, то алгебру $A(X)$ называют алгеброй Дирихле. Алгебра $A(X)$ унимодулярна, если линейные комбинации унимодулярных функций из $A(X)$ плотны в $A(X)$.

Пусть $M-$ множество всех линейных мультипликативных функционалов на $A(X)$. Говорят, что $m_{1}, m_{2} \in M$ принадлежат одной и той же доле Глисона, если $d\left(m_{1}, m_{2}\right)=\left\|m_{1}-m_{2}\right\|<2$. Принадлежность к одной и той же доле Глисона есть отношение эквивалентности на $M$. Согласно теореме Вермера (см. $[1,2])$, если $G \subset M$ - неодноточечная доля Глисона алгебры Дирихле $A(X)$, то существует гомеоморфизм $\varphi: D^{0} \rightarrow G$ аналитического диска $D^{0}=\{z \in \mathbb{C}:|z|<1\}$ на $G$, такой, что преобразование Гельфанда $\hat{f}(z)=\varphi(z)(f)$ для любого $f \in$ $A(X)$ есть аналитическая функция.

Следует отметить, что пример равномерной алгебры, не имеющей неодноточечных долей, такой, что каждая точка из пространства ее максимальных идеалов является точкой пика, был построен Коулом [3]. Бэсенер [4] предложил более конкретный пример равномерной алгебры, определенной на компактном метрическом пространстве (пространстве ее максимальных идеалов) со следующими свойствами: она не совпадает с множеством всех непрерывных функций на пространстве ее максимальных идеалов и каждая точка пространства максимальных идеалов есть точка пика.

В данной работе строится пример максимальной унимодулярной алгебры Дирихле, одноточечные доли Глисона которой плотны в множестве всех линейных мультипликативных функционалов в *-слабой топологии.

1. Алгебра Бляшке. Пусть $S=\{z \in \mathbb{C}:|z|=1\}$ - единичная окружность и $D=\{z \in \mathbb{C}:|z| \leqslant 1\}$ - единичный диск в $\mathbb{C}$. Бесконечное семейство $\left\{b_{k}(z)\right\}_{k=1}^{\infty}$ конечных произведений Бляшке

$$
b_{k}(z)=e^{i \theta_{k}} \prod_{j=1}^{n_{k}} \frac{z-z_{j, k}}{1-\bar{z}_{j, k} \cdot z}, \quad 0 \leqslant\left|z_{j, k}\right|<1,0 \leqslant \theta_{k}<2 \pi,
$$

порождает проективный предел

$$
D_{1} \stackrel{b_{1}}{\longleftarrow} D_{2} \stackrel{b_{2}}{\longleftarrow} D_{3} \stackrel{b_{3}}{\longleftarrow} \cdots
$$

единичных дисков $D_{k}=D$, где $b_{k}: z \rightarrow b_{k}(z)$. Сужение этой последовательности на единичные окружности $S_{k}=S$ образует проективный предел

$$
S_{1} \stackrel{b_{1}}{\longleftarrow} S_{2} \stackrel{b_{2}}{\longleftarrow} S_{3} \stackrel{b_{3}}{\longleftarrow} \cdots
$$

единичных окружностей. Пусть

$$
D_{\infty}=\varliminf_{k}^{\lim } D_{k}, \quad S_{\infty}=\varliminf_{k}^{\lim } S_{k} .
$$


Отметим, что $b_{k}\left(z_{k+1}\right)=z_{k}$ и суперпозиция $b_{k}^{\infty}=b_{k} \circ b_{k+1} \circ b_{k+2} \circ \cdots$ порождает проекцию $b_{k}^{\infty}: D_{\infty} \rightarrow D_{k}$, причем $b_{k}^{\infty}=b_{k} \circ b_{k+1}^{\infty}, b_{k}^{\infty}\left(S_{\infty}\right)=S$.

Пусть $A_{k}, k=1,2,3, \ldots$, - замкнутая в sup-норме подалгебра алгебры $C\left(D_{\infty}\right)$, порожденная единичным элементом алгебры $C\left(D_{\infty}\right)$ и функцией $b_{k}^{\infty}$. Из (1) следует, что

$$
b_{k}^{\infty}=b_{k}\left(b_{k+1}^{\infty}\right)=e^{i \theta_{k}} \prod_{j=1}^{n_{k}} \frac{b_{k+1}^{\infty}-z_{j, k}}{1-\bar{z}_{j, k} \cdot b_{k+1}^{\infty}} .
$$

Пусть

$$
A=\bigcup_{k=1}^{\infty} A_{k}
$$

ОПРЕДЕЛЕНИЕ 1. Алгеброй Бляшке, образованной произведениями Бляшке $\left\{b_{k}(z)\right\}_{k=1}^{\infty}$, называется равномерная алгебра $B$ на $D_{\infty}$, порожденная функциями $\left\{b_{k}^{\infty}\right\}_{k=1}^{\infty}$, т. е. $B=\bar{A}$.

В работе [5] доказана следующая теорема.

Теорема 2. (i) Пространство линейнъх мультипликативных функционалов на алгебре $B$ совпадает с $D_{\infty}$.

(ii) Граница Шилова этой алгебры есть $S_{\infty}$.

(iii) Сужение алгебры $B$ на $S_{\infty}$ есть унимодулярная максимальная алгебра Дирихле.

2. Доли Глисона алгебры $\boldsymbol{B}$. Каждое произведение Бляшке $b(z)$ порождает накрытие $b: D \rightarrow D, z \rightarrow b(z)$. Точка $z_{0} \in D^{0}=\{z \in \mathbb{C}:|z|<1\}$ называется точкой разветвления для накрытия $b: D \rightarrow D$, если у этой точки нет окрестности $U$, такой, что сужение накрытия $b$ на $U$ инъективно. В этом случае можно подобрать такую окрестность $U$, чтобы сужение накрытия $b$ на $U$ было биголоморфно отображению $z^{k}: D \rightarrow D, z \rightarrow z^{k}$ с $k>1$ (см. [6, с. 43$44])$. Число $k=\operatorname{deg}_{b}\left(z_{0}\right)$ называется степенью разветвления точки $z_{0}$. Согласно предложению 2.1 из [4, р. 15], если $f_{1}(z)$ и $f_{2}(z)$ - аналитические функции на $D^{0}$ и $z_{1}=f_{2}\left(z_{0}\right) \in D^{0}$, то

$$
\operatorname{deg}_{f_{1} \circ f_{2}}\left(z_{0}\right)=\operatorname{deg}_{f_{1}}\left(z_{1}\right) \cdot \operatorname{deg}_{f_{2}}\left(z_{0}\right) .
$$

ОПРЕДЕЛЕНИЕ 3 . Точка $s \in D_{\infty}, s=\left(z_{1}, z_{2}, \ldots, z_{k}, \ldots\right), b_{k}\left(z_{k+1}\right)=z_{k}$, называется особой точкой в $D_{\infty}$, если для любого натурального числа $n$ найдется целое число $k>n$, такое, что точка $z_{k+1}$ является точкой разветвления для накрытия $b_{k}: D_{k+1} \rightarrow D_{k}$, т. е. $\operatorname{deg}_{b_{k}}\left(z_{k+1}\right) \geqslant 2$.

Для долей Глисона и определенных таким образом особых точек из $D_{\infty}$ справедлива следующая теорема.

ТЕОремА 4. Каждая доля Глисона, содержащая особую точку, есть одноточечное множество.

3. Пример. Пусть $z_{1} \in D^{0}$ - точка разветвления для накрытия $b_{1}: D \rightarrow D$, порожденного произведением Бляшке $b_{1}(z)$. Если $z_{0} \in b_{2}^{-1}\left(z_{1}\right)$, где $b_{2}(z)-$ произведение Бляшке, то $z_{0}$ есть точка разветвления для накрытия $b_{1} \circ b_{2}: D \rightarrow D$. Действительно,

$$
\operatorname{deg}_{b_{1} \circ b_{2}}\left(z_{0}\right)=\operatorname{deg}_{b_{1}}\left(z_{1}\right) \cdot \operatorname{deg}_{b_{2}}\left(z_{0}\right) \geqslant 2, \quad \text { так каK } \operatorname{deg}_{b_{1}}\left(z_{1}\right) \geqslant 2 .
$$


Данный факт можно применить для доказательства следующей леммы.

Лемма 5. Для любых точек $w_{1}, \ldots, w_{n} \in D^{0}$ найдется конечное произведение Бляшке $b(z)$, такое, что для всех $k=1, \ldots, n$ прообраз $b^{-1}\left(w_{k}\right)$ содержит хотя бы одну точку разветвления для накрытия $b: D \rightarrow D, z \rightarrow b(z)$.

Известно (см. [7, с. 190]), что пространство мультипликативных функционалов диск-алгебры $A(D)$ есть $D$ и база *-слабой топологии на $D$ задается множествами вида $\left\{z \in D:\left|z-z_{0}\right|<\varepsilon\right\}$. Аналогично, за базу *-слабой топологии точки $s_{0} \in D_{\infty}$ можно взять множества вида $\left\{s \in D_{\infty}:\left|b_{k}^{\infty}(s)-b_{k}^{\infty}\left(s_{0}\right)\right|<\varepsilon\right\}$, $\varepsilon>0, k=1,2, \ldots$.

Используя предыдущую лемму, можно сформулировать следующую теорему:

Теорема 6. Существует алгебра Бляике, одноточечные доли Глисона которой плотны в $D_{\infty}$ в ${ }^{*}$-слабой топологии.

Из теорем 2, 4 и 6 непосредственно следует главный результат.

Теорема 7. Существует максимальная унимодулярная алгебра Дирихле, у которой одноточечные доли Глисона плотны в пространстве мультипликативных функционалов.

\section{ЛитерАтУра}

1. Wermer J. J. Duke Math., 27, 373-382 (1960). 2. Гамелин T. Равномерные алгебры. Мир, М., 1973. 3. Browder A. Introduction to function algebras. Benjamin, New York, 1969. 4. Basener W. G. Trans. Amer. Math. Soc., 182, 353-381 (1973). 5. Grigoryan S. A., Tonev T. V. Internat. J. Math. Math. Sci., 27, No. 10, 599-620 (2001). 6. Форстер О. Римановы поверхности. Мир, М., 1980. 7. Гарнетm Дж. Ограниченные аналитические функции. Мир, М., 1984.

Казанский государственный энергетический университет e-mail: Evgene.Yashagin@ksu.ru

Поступило в редакцию 31 мая 2004 г. 